\title{
Antecedents of Constructive Human-AI Collaboration: An Exploration of Human Actors' Key Competencies
}

\author{
Thomas Süße, Maria Kobert, and Caroline Kries \\ Bielefeld University of Applied Sciences, Faculty of Engineering and Mathematics, \\ Gütersloh Campus, Langer Weg 9 a \\ 33332 Gütersloh, Germany
}

\begin{abstract}
Artificial intelligence (AI) has become an integral element of modern machines, devices and materials, and already transforms the way humans interact with technology in business and society. The traditionally more hierarchical interaction, where humans usually control machines, is constantly blurring as machines become more capable of bringing in their own (intelligent) initiatives to the interaction with humans. Thus, nowadays it is more appropriate to consider the interactive processes between humans and machines as a novel form of interdependent learning efforts between both sides, where processes such as critical discourses between humans and machines may take place (hybrid intelligence). However, these developments demand a shift in the understanding about the role of technology at work and about specific competencies required among human actors to collaborate constructively and sustainably with AI systems. This paper seeks to address this issue by identifying human actors' key competencies, which enable a more constructive collaboration between humans and intelligent technologies at work.
\end{abstract}

Keywords: artificial intelligence, human-AI collaboration, AI-related competencies

\section{Introduction}

The opportunities and capabilities of interconnected intelligent technologies foster the further emergence of smart and sustainable collaborative networks and enable more integrative forms of interaction as well as resource, knowledge or information exchange between a greater variety of heterogeneous actors and artificial agents perceived as increasingly intelligent. These developments also result in changes on the micro level of human-machine interaction and directly affect the way work processes are performed. Interconnected agents based on artificial intelligence (AI) in modern work environments, for instance, increasingly assist human actors by taking on standardized tasks and recently also tasks of limited complexity to support or enable more collaborative communication, information, decision-making or production processes [1-4]. Some typical applications are chatbots that take over routine interactions with customers [5], algorithms that support the selection of new employees [6], medical diagnoses [7, 8], predictive maintenance of machines [9] or intelligent robots that are able to work safely alongside humans in factories, where they take on highly repetitive and exhausting tasks [9]. The company Hyundai, for example, recently introduced wearable robotic devices which can adapt quickly to various tasks, contexts and 
workers in order to perform jobs "with superhuman endurance and strength" [10]. Moreover, such intelligent agents or machines can be trained by employees during the working process $[3,5,9,11]$. Thus, the human can be regarded as a trainer or educator of machines and the latter as a human's trainee. As soon as these machines are sufficiently trained, they can possibly take on the role of the trainer for new or inexperienced colleagues. Thus, the roles between human and machine are becoming more interchangeable in modern work environments $[3,9]$.

Based on these examples, it is evident that intelligent technologies are transforming traditional human-machine interaction. The ability of AI-enabled technology to learn independently, interpret context, make its own decisions and communicate these to humans and, thus, influence human decision-making and actions ensures that it is viewed by humans increasingly as a colleague, teammate or even a buddy $[1,3,9,12]$. Though these developments are currently in their early stages and machines' rudimentary intelligent capabilities are only partially comparable to those of humans, an understanding towards a human-AI collaboration has evolved $[1,2,8,13]$. On the one hand obvious benefits are seen in achieving higher efficiency and quality by humans and machines complementing each other [3,14], on the other hand the absence of acceptance and understanding on the part of employees towards the new technology, entails new challenges [15].

One important step to overcome these challenges is seen in further training and development of employees' competencies [16]. Consequently, the question of which competencies humans need or will need in the future to be able to work effectively and sustainably with AI-based technologies arises $[9,11]$. In practice, it has already been shown that AI-related competencies of employees represent a particularly critical success factor for the actual unfolding of smart technologies' potentials [11, 17-19]. However, so far, particularly IT, mathematical, leadership and social skills have been discussed as essential elements or partial aspects of AI skills in current research [20]. Furthermore, occupational fields were mainly examined in which predominantly academics are active, but employees who collaborate with AI systems in the workplace and are only indirectly involved in the introduction or basic development of AI have so far received less attention [13, 21]. However, intelligent technologies are increasingly present across many working areas - from production to administration or social professions $[11,19]$.

The objective of this work is to explore and systematize the competencies humans working in different industry sectors and positions may need to not only be able to introduce, monitor or use but rather to constructively collaborate with more intelligent technologies at work. To derive the competencies we applied an iterative and reflexive approach. We performed literature research on recent technical and social developments as well as challenges in the field of human-AI collaboration. We validated and refined the findings in workshops and ongoing conversations with experts on human-AI applications from science and business. In this article, the final findings are presented.

We consider the systematization of key competencies as an important initial step in the derivation of an AI-related competencies framework. The framework shall provide an important contribution to a more specific understanding in science and business about the key enablers for human-AI collaboration and mutual learning between human and technology at work. 


\section{Related Work and Conceptual Background}

The term "artificial intelligence (AI)" was first coined by John McCarthy in 1956 [22] and is still not clearly defined. A very popular definition from Nils J. Nilsson [23] reads: "Artificial Intelligence is that activity devoted to making machines intelligent, and intelligence is that quality that enables an entity to function appropriately and with foresight in its environment." Kaplan and Haenlein [15] state that AI is "a system's ability to correctly interpret external data, to learn from such data, and to use those learnings to achieve specific goals and tasks through flexible adaptation." According to the first definition, AI has the potential to be comparable or even superior to human intelligence [14]. However, up to now, scientists have outlined that a so-called strong or general AI, which can be applied to any problem and is, therefore, comparable to human intelligence, does not exist yet but is an important issue in current research [24]. Furthermore, the so-called super AI, which is even supposed to be superior to human intelligence, is so far only a philosophical speculation [25]. Thus, we argue that the definition given above by Kaplan and Haenlein [15] describes the current perception and understanding about existing and widely used AI as narrow AI more appropriately. In fact, narrow AI is defined as insentient and typically focused on a narrow, very specific task or area of applications [26].

Today's most common narrow AI technique is machine learning or, more precisely, a class of it, the so-called deep learning. As the name already indicates, deep learning neural networks learn from data by extracting complex structures and creating calculation models. These are composed of several processing layers and can thus create different levels of abstraction [27]. With this technique, intelligent machines are able to understand written or spoken language, pictures or videos, to draw conclusions on this basis independently as well as interact or communicate with their environment [26]. The latest developments even show that robots equipped with such AI techniques are able to detect, respond and display emotions [28].

Even though, as already indicated, today's intelligent machines are based on weak $\mathrm{AI}$ and are far from being as intelligent as humans, they tend to ascribe certain 'human traits' to an AI system. As a result, the interaction between humans and AI-based agents can transform itself into a collaboration and cooperation 'at eye level'. Thus, the AIrelated technological enhancements and their dissemination contribute to the emergence of new forms of human-machine interaction. Whereas previously it was primarily the human being who took the initiative and largely specified what the machine had to work on, this hierarchical relationship is increasingly being transformed by AI-related capabilities. As AI-enabled agents become increasingly capable of making their own decisions, performing simple or repetitive tasks and improving more independently over time $[9,11]$, these agents are constantly developing towards artificial colleagues or teammates for their human counterparts at work [1, 12]. As a result, human-machine interaction is transforming to a more integrative level, the human-AI collaboration $[1,3,13]$. In essence, this means that AI and humans will work and learn increasingly 'hand-in-hand' and mutually integrative in the future to achieve higher levels of performance $[1,3,29]$. This is what we refer to as constructive humanAI collaboration. In the context of human-AI collaboration, the term hybrid intelligence has also recently been introduced [2], which is defined as the combination of artificial 
and human intelligence. The benefit of the integration of the latter is seen in the capability of solving increasingly complex problems faster and more efficiently than if only one of them had been involved. A central aspect of hybrid intelligence is that over time, not only the socio-technical system as a whole but also the human and the machine themselves improve through the experiences gained in solving problems collaboratively and by learning in a mutual beneficial way [2].

In practice, it has already been shown that a closer collaboration between humans and technology can be very beneficial $[3,9]$ for example, humans can be released from dangerous, hazardous, physically demanding or monotone tasks and are, therefore, enabled to take over more cognitively challenging or creative tasks $[3,11]$. Nevertheless, from employees' point of view there are fundamental reservations and challenges, such as the fear of being replaced by the machine teammate or the lack of transparency and understanding about its functionality, preventing the new technology's further dissemination. Many attempts to overcome these problems focus on the design of AI-systems to be more explainable, transparent or applicable to humans $[30,31]$. However, holistic approaches focusing on the human counterpart are also required. One main aspect in this context is that job profiles, roles and working conditions are changing [15]. For example, humans will rather have to train machines in order to enable them to take over repetitive tasks or understand and explain technology's decisions or outcomes [3,9]. Thus, the aim is to enable employees to take on new or 'higher-quality' tasks, on the one hand, and to work together with AI systems more effectively, responsibly and sustainably, on the other hand $[1,3,11]$. This leads to the question about which specific competencies are indispensable for a constructive and responsible human-AI collaboration [26, 32]. Research to date has revealed that an essential prerequisite for a successful introduction and sustainable performance of AIbased systems can be seen in the existence of specific human actors' competencies for collaborating with AI technologies [11, 19]. However, further systematizations and conceptualizations of the competencies demanded are still rare and fragmented.

We refer in this article to competencies as behavioral repertoires that people carry out at the job, for example, coping with job-related tasks [33]. This means that competencies should not be mixed up with performance outcomes, such as effectivity or quality, but should, instead, be considered as crucial enablers for successful job performance. Consequently, we refer to competencies as actual "dimensions of behavior which are related to superior job performance" [33]. These behavioral dimensions of human actors' competencies can further be systematized by the three interdependent clusters of cognitive, emotional and social competencies [34]. Cognitive competencies include elements such as systems thinking or pattern recognition. Emotional competencies refer to self-awareness and self-management or emotional self-control. Social competencies include elements such as social awareness or relationship management, for example, empathy, teamwork or inspiration. We find this systematization of competencies, which refers explicitly to the outstanding performance of human actors at work, highly applicable for the development of a framework of competencies demanded for human-AI collaboration, as we showed above that AI-based systems trigger profound changes in work processes and human job roles. 


\section{Towards a Framework of AI-specific Demands of Human Actors' Competencies}

In the following, a set of human actors' competencies supporting constructive humanAI collaboration is presented. These competencies were identified by an extensive literature research on the latest developments as well as challenges in intelligent technologies' application areas. The research was complemented with ongoing dialogues with experts applying or implementing AI-based systems.

There are already some very fruitful AI-related investigations about competence and skill demands in literature. The conceptualization of "AI literacy" introduced by Long and Magerko [21] holds 17 skills that humans need to work effectively and sustainably with and critically evaluate AI technologies. Another study by Pfeifer [35] outlines the potential of applying AI in the context of predictive maintenance and consulting-intensive clerical work. An important conclusion from this study is that employees have to understand the potentials and limitations of AI-based systems and should be able to place them and their results in the professional context as well as the situational requirements in order to quickly exploit the potential of the technology in the company [35]. A further examination of job advertisements for the fields of data science, software and business development, and sales, combined with a literature review, reveals that technical competence, leadership and soft skills are required for the usage of AI [20].

These works focus predominantly on the introduction of intelligent technologies in a narrow field of work environments and on higher qualified positions. Namely, the identified competencies are rather basic or technical and may be applied to humans developing and supervising AI-systems, rather than to humans who are actually collaborating with such systems. However, in the near future a penetration of various employment fields with more interactive AI technologies is expected. Thus, while previous research provides a great basis for further systematization of AI-related human competencies, there is a lack of a sufficient and holistic behavioral framework about human actors' competencies demanded for a constructive human-AI collaboration in organizations. The framework presented below shall provide an important starting point for closing this gap.

During our research we found it fruitful and highly applicable to refer to the clustering dimensions of cognitive, emotional and social competencies [34] in order to provide a more comprehensive and systematic framework. Based on our analysis and investigations, nine distinct dimensions of human actors' competencies are revealed to be essential elements of an AI competencies framework (see Table 1). We label these dimensions as: (1) Context-specific understanding and interpretation of AI impulses; (2) expressing oneself comprehensibly towards the AI colleague; (3) evaluating the intelligence and capabilities of AI agents; (4) dealing with AI systems in a reflective manner; (5) engaging oneself in a constantly constructive discourse; (6) handling sensitive data critically; (7) constantly upholding ethical and moral standards; (8) showing an awareness of an AI agent as a sort of virtual colleague; and, finally, (9) negotiating one's own recovery phases with AI agents. Table 1 shows a clustering of these nine dimensions allocated to the three clusters cognitive, emotional and social. 
We are aware that a clear allocation of all AI-related competencies to one unique cluster is rather challenging. Thus, we marked only that cluster for each AI-related competence with an " $x$ " which we find shows the strongest suitability based on our investigations.

Table 1: Clustering of AI-related competencies (AI competencies framework)

\begin{tabular}{|l|c|c|c|}
\hline & \multicolumn{2}{c|}{ Clustering } \\
\hline AI-related competencies & cognitive & emotional & social \\
\hline 1. Context-specific understanding and interpretation of AI impulses & $\mathrm{X}$ & & \\
\hline 2. Expressing oneself comprehensibly towards the AI colleague & & & $\mathrm{x}$ \\
\hline 3. Evaluating the intelligence and capabilities of AI agents & $\mathrm{x}$ & & \\
\hline 4. Dealing with AI systems in a reflective manner & $\mathrm{x}$ & & \\
\hline 5. Engaging oneself in a constantly constructive discourse & & $\mathrm{x}$ \\
\hline 6. Handling sensitive data critically & $\mathrm{x}$ & \\
\hline 7. Constantly upholding ethical and moral standards & & $\mathrm{x}$ \\
\hline 8. Showing an awareness of an AI agent as a sort of virtual colleague & & $\mathrm{x}$ & \\
\hline 9. Negotiating one's own recovery phases with AI agents & & $\mathrm{x}$ & \\
\hline
\end{tabular}

The nine AI-related competencies can be described in more detail as follows.

\section{Context-specific understanding and interpretation of AI impulses}

One major challenge in human-AI collaboration is that humans often cannot understand how or why an AI system arrives at a specific conclusion or decision. Clinical decision support systems that are able to, for example, detect cancer and assist clinicians to inspect clinical cases are difficult to integrate into practice because clinicians do not understand how their potential machine teammates draw their conclusions and make decisions and, thus, have difficulty trusting them [8]. This circumstance is often referred to as a 'black box' in science [31]. There have already been attempts in research to overcome the AI's black box system by developing explainable AI (XAI); this should be more transparent or able to explain or justify its behaviors and decisions $[12,31,36]$. However, in such cases where systems are based on complex deep neural networks, not even data scientists or software developers have yet been able to consistently understand the underlying decision rules [37]. Nevertheless, an essential demand to be able to work constructively with intelligent systems, to use them as a basis for one's own decisions and actions, or to explain their conclusions to others is that humans have to be able to understand and interpret AI-based technology's decisions and impulses regarding the specific context.

2. Expressing oneself comprehensibly towards the AI colleague

From the perspective of the AI-based learning algorithms, researchers and practitioners have revealed that a common challenge is that AI often fails to understand humans as well. This is particularly known from communicating with virtual assistants, which frequently tend to misunderstand human actors due to various reasons, such as different perceptions or interpretations of constructs, dynamic situations or ambiguous problems. There is a prominent example for Apple's Siri, which took the request "Call me an ambulance!" as a request to name its user "Anne Ambulance" [38]. It is also important in working situations, for example, to receive certain information from the AI system or give it an instruction, that the AI-based agent understands and interprets the request correctly in order to initiate appropriate actions [3]. Thus, humans have to be able to express themselves in a way that is comprehensible for the technology. This 
contributes to the (re)creation of a shared meaning of key concepts between human and AI.

\section{Evaluating the intelligence and capabilities of AI agents}

Humans can also tend to place too much confidence in AI technology or rely completely on its judgment because AI can be very good in some specific areas and can even outperform humans in speed, scalability and quantitative capabilities [14, 28]. Instead, humans should know how to combine their distinctive human skills with those of a smart technology [3]. To be more precise, intelligent machines are, for example, able to generate new combinations, classify things or take over repetitive activities. Humans, as counterparts, can come up with unique or original ideas, be emphatic or bring in human judgement $[1,14]$. When building a vehicle, for example, an AI-based machine can take over simple, repetitive tasks, such as checking parts for quality or lifting heavy parts, but, in some cases, it is still more challenging for machine-like robots to perform dexterity tasks, such as assembling a gear motor [3]. The AI-based systems are usually trained for a specific purpose using data currently available and not necessarily covering all possible cases. Exemplarily, an AI-medical device was recently developed to predict the risk of cardiovascular heart disease [7]. However, the clinician who collaborates with this technology has to be aware that there is a risk that it could draw inaccurate predictions for patients of various ethnicities based on the fact that this AI system was trained with patients' data from three hospitals in a particular geographical area in Germany [7]. Moreover, AI systems are usually not able to transfer or generalize their ability or knowledge as easily as humans do [15]. Each new task requires a lot of data and extensive training. Consequently, it might not be immediately possible to teach an AI that has been trained to visually examine pictures or objects to recognize noises [9]. If the demands for AI agents change over time, then retraining might be required with the help of appropriate training data [39]. Thus, it is necessary for humans to be able to evaluate the suitability of an AI agent's capabilities concerning the execution of specific tasks and the complementarity to the human counterparts.

\section{Dealing with AI systems in a reflective manner}

As has already been indicated, nearly all AI algorithms are based on statistical or probabilistic methods. Therefore, it is usually impossible to train them to be $100 \%$ accurate and, thus, they can, similar to humans, make errors in specific cases [39]. This can be illustrated by an incident in Australia in 2016 where an AI-based system assigned with verifying the legality of social benefit payments was introduced. The technology detected those cases wrongly receiving unemployment benefits or social assistance and then automatically sent reminder letters to the relevant people. There was, in fact, no basis for the refunds claimed by it in 7,000 of 200,000 dunning letters sent by the software [40]. To prevent such incidents from happening, humans have to deal more reflectively and critically with the AI agent's conclusions or suggestions to be able to identify possible errors or incorrect decisions before they can harm or affect other people. In fact, this competence is an important prerequisite for constructively providing the AI-based technology with appropriate feedback that is comprehensible to it so that it can learn from mistakes and improve over time [40]. Such evolutionary progression is hardly possible if the system, for example, receives exclusively onesided feedback, e.g. no feed-back regarding the fact that an individual denied credit was actually able to repay [40]. 


\section{Engaging oneself in a constantly constructive discourse}

Researchers and practitioners argue that learning processes should take place in both directions, from the AI agent to the human being and vice versa. The humans, for example, can receive feedback from their digital assistant on how a specific task was conducted regarding quality or performance indicators. This provides humans with more specific information where and possibly how to improve their own work processes or competencies [1,2]. Regarding a constructive collaboration with AI systems in the workplace, this means that employees must be able to constructively absorb the impulses of the AI system and interpret them as support for their own ongoing learning processes and constant improvement. Consequently, humans should be more aware of an AI agent in its role as a helpful sparring partner in critical situations, during task performance or problem solving processes $[14,40]$. Thus, a key demand of humans' competencies can be seen in behavioral patterns of constantly constructive discourse and dialogue between them and the AI.

\section{Handling sensitive data critically}

It is well known that most AI algorithms are based on personal and, therefore, often highly sensitive private data. Chatbots in customer care, for instance, are trained using personal data of customers. Search engines, recommender systems or digital assistants learn constantly from humans who engage with them [42]. The AI agents in production systems are very often based on data of high economic value for business organizations. Although the goal of intelligent technologies is to provide their human counterpart with optimal and personalized services, critical data gathering can compromise privacy, anger customers, endanger the competitive advantages of businesses and run afoul of the law [3]. Therefore, a further important competence demand for humans is to be able to handle sensitive data critically and with all required caution [42, 43].

\section{Upholding ethical and moral standards}

In 2014, a hiring AI system, which has been assigned to select suitable applicants for technical jobs, was developed and introduced by Amazon. However, it preferred male applicants over women, since, based on the data it was given, it learned that more men than women work in technological fields [44]. One can see from this example and many others, e.g. credit approving AI agents, which are known to be able to discriminate against people in certain groups $[3,38]$ that AI systems also have the potential to make biased decisions. This circumstance causes many controversies in society and there have already been attempts in politics and science to overcome this problem. Some governments, for example, have introduced principles and guidelines for AI developers [45]. Thus, AI researchers are working on developing trustworthy AI (TAI) that should be unbiased and just $[7,46]$. However, because, in practice, AI systems are increasingly learning from direct interaction with humans, orientation to ethical and moral standards and values represent crucial requirements for humans who constantly collaborate with AI systems [40, 47].

\section{Showing an awareness of an AI agent as a sort of virtual colleague}

Human-AI collaboration can also be particularly challenging if the AI only has a virtual appearance, on the one hand, but, on the other hand, is to be attributed traits of a great teammate, for example, for joint task processing in stressful situations [28, 48].

9. Negotiating one's own recovery phases with $\mathrm{AI}$ agents

Finally yet importantly, it has to be considered that AI systems, in comparison to humans, require no or possibly other types of recovery phases. This needs to be 
negotiated and taken into account in the future between AI agents and humans in a similar way as between humans. The humans should be highly aware of their own physical and psychological limitations and must interpret signs of exhaustion correctly in order to insist on recovery phases and detachment from work [49] .

\section{Conclusion and Outlook}

Our results reveal that the competencies demanded from the AI-based agent's counterpart, the human actor, cannot be covered completely by IT and mathematical skills, context competencies or leadership and social competencies. We suggest, instead, that there are additional cognitive, emotional and social competencies of humans that should be considered to enable a constructive human-AI collaboration. Our framework, shown in Table 1, introduces nine key competencies, which can be regarded as rather independent of specific tasks or problem situations at work but should be understood as general behavioral patterns that humans might be able to perform or learn when collaborating with AI-based agents at work. It also shows that there are certain interrelationships between the nine competencies identified so far; for example, competencies of dealing with AI in a reflective manner (4) might be interrelated with competencies of handling sensitive data critically (6) or competencies of upholding ethical and moral standards (7). In addition, we assume that there might be rather context and job specific configurational types of the set of introduced competencies (compositions) showing different levels of importance or relevance of each competence dimension in relation to the other competencies identified.

Thus, we expect that specific configurations or compositions of these AI-related competencies might enable a greater number of employees from various fields and professions to collaborate more constructively with AI. Furthermore, we argue that human actors holding these competencies will be able to participate and engage more constructively in the further development of AI-based systems as AI development should not only be a question of appropriate techniques and algorithms but has to deal much more with the transformational changes of work and society.

Concerning the framework deduced, we seek to initiate and contribute to further discussion and progress regarding the understanding and knowledge about transforming work environments, the changing role of employees and, consequently, the shift of job demands and challenges for the human actor in increasingly collaborative and interconnected work environments.

We invite future research to take up this framework in order to develop it further towards a scientifically sound model of human's AI-related competencies. Therefore, more qualitative and quantitative empirical field research seems to be necessary to evaluate, validate and adjust the results produced by our research. We argue that an empirically evaluated AI competence model is a key prerequisite for specific competence evaluation and development of humans within future work environments. This may also mean that in areas such as human resource management, researchers and practitioners should better understand the distinct capabilities and types of interaction of various AI-based agents in order to relate those to challenges for human actors during 
collaborative processes. Thus, research and practice may benefit from more interdisciplinary research and development approaches in this area.

\section{References}

1. Seeber, I., Bittner, E., Briggs, R.O., de Vreede, G.-J., de Vreede, T., Druckenmiller, D., Merz, A.B., Oeste-Reiß, S., Randrup, N., Schwabe, G., Söllner, M.: Machines as Teammates: A Collaboration Research Agenda. 10 (2018). https://doi.org/10.24251/HICSS.2018.055.

2. Dellermann, D., Calma, A., Lipusch, N., Weber, T., Weigel, S., Ebel, P.: The Future of Human-AI Collaboration: A Taxonomy of Design Knowledge for Hybrid Intelligence Systems. Presented at the Hawaii International Conference on System Sciences (2019). https://doi.org/10.24251/HICSS.2019.034.

3. Wilson, H.J., Daugherty, P.R.: Collaborative intelligence: humans and AI are joining forces. Harvard Business Review. 96, 114-123 (2018).

4. Süße, T.: Digital Citizenship Behavior in Organization as Indicator for Actors' Co-creative Problem-Solving in Ecosystem-Oriented Work Environments. In: Ahram, T., Taiar, R., Colson, S., and Choplin, A. (eds.) Human Interaction and Emerging Technologies. pp. 675681. Springer International Publishing, Cham (2020).

5. Hildesheim, W., Michelsen, D.: Künstliche Intelligenz im Jahr 2018 - Aktueller Stand von branchenübergreifenden KI-Lösungen: Was ist möglich? Was nicht? Beispiele und Empfehlungen. Künstliche Intelligenz. 119-142 (2019). https://doi.org/10.1007/978-3662-57568-0_8.

6. Upadhyay, A.K., Khandelwal, K.: Applying artificial intelligence: implications for recruitment. SHR. 17, 255-258 (2018). https://doi.org/10.1108/SHR-07-2018-0051.

7. Zicari, R.V., Brodersen, J., Brusseau, J., Dudder, B., Eichhorn, T., Ivanov, T., Kararigas, G., Kringen, P., McCullough, M., Moslein, F., Mushtaq, N., Roig, G., Sturtz, N., Tolle, K., Tithi, J.J., van Halem, I., Westerlund, M.: Z-Inspection $®$ : A Process to Assess Trustworthy AI. IEEE Trans. Technol. Soc. 1-1 (2021). https://doi.org/10.1109/TTS.2021.3066209.

8. Cai, C.J., Winter, S., Steiner, D., Wilcox, L., Terry, M.: "Hello AI": Uncovering the Onboarding Needs of Medical Practitioners for Human-AI Collaborative Decision-Making. Proc. ACM Hum.-Comput. Interact. 3, 1-24 (2019). https://doi.org/10.1145/3359206.

9. Ahlborn, K., Bachmann, G., Biegel, F., Bienert, J., Falk, S., Fay, A., Gamer, T., Garrels, K., Grotepass, J., Heindl, A.: Technologieszenario „Künstliche Intelligenz in der Industrie 4.0 “. Bundesministerium für Wirtschaft und Energie (BMWi). (2019).

10. A light-weight passive upper arm assistive exoskeleton based on multi-linkage springenergy dissipation mechanism for overhead tasks. Robotics and Autonomous Systems. 122, 103309 (2019). https://doi.org/10.1016/j.robot.2019.103309.

11. PLS - Plattform Lernende Systeme: Arbeit, Qualifizierung und Mensch-MaschineInteraktion. acatech Deutsche Akademie der Technikwissenschaften, München (2019).

12. Wanner, J., Herm, L.-V., Janiesch, C.: Countering the Fear of Black-boxed AI in Maintenance: Towards a Smart Colleague. 8 (2019).

13. Wang, D., Weisz, J.D., Muller, M., Ram, P., Geyer, W., Dugan, C., Tausczik, Y., Samulowitz, H., Gray, A.: Human-AI Collaboration in Data Science: Exploring Data Scientists' Perceptions of Automated AI. Proc. ACM Hum.-Comput. Interact. 3, 1-24 (2019). https://doi.org/10.1145/3359313.

14. Othman, A., Eriksson, Y., Chirumalla, K.: Mapping Artificial Intelligence (AI) Capabilities around Human Competences: An explorative study. 82. 
15. Kaplan, A., Haenlein, M.: Siri, Siri, in my hand: Who's the fairest in the land? On the interpretations, illustrations, and implications of artificial intelligence. Business Horizons. 62, 15-25 (2019). https://doi.org/10.1016/j.bushor.2018.08.004.

16. Weber, D.M.: Künstliche Intelligenz Wirtschaftliche Bedeutung, gesellschaftliche Herausforderungen, menschliche Verantwortung. Bitkom e. V., DFKI, Berlin, Kaiserslautern (2017).

17. Bundesministeriums für Arbeit und Soziales (BMAS): Begleitband des Bundesministeriums für Arbeit und Soziales. 356 (2020).

18. Wangermann, T.: KI in KMU: Rahmenbedingungen für den Transfer von KIAnwendungen in kleine und mittlere Unternehmen. Konrad Adenauer Stiftung (2020).

19. Rammer, C., Bertschek, I., Schuck, B., Demary, V., Goecke, H.: Einsatz von Künstlicher Intelligenz in der Deutschen Wirtschaft: Stand der KI-Nutzung im Jahr 2019. ZEWGutachten und Forschungsberichte (2020).

20. Anton, E., Behne, A., Teuteberg, F.: The human behind artificial intelligence - An operationalisation of AI competencies. In: In Proceedings of the 28th European Conference on Information Systems (ECIS). pp. 19-36 (2020). https://aisel.aisnet.org/ecis2020_rp/141.

21. Long, D., Magerko, B.: What is AI Literacy? Competencies and Design Considerations. In: Proceedings of the 2020 CHI Conference on Human Factors in Computing Systems. pp. 116. ACM, Honolulu HI USA (2020). https://doi.org/10.1145/3313831.3376727.

22. Ashby, W.R., Culbertson, J.T., Davis, M.D., Kleene, S.C., De Leeuw, K., Mac Kay, D.M., Mc Carthy, J., Minsky, M.L., Moore, E.F., Shannon, C.E., Shapiro, N., Uttley, A.M., Von Neumann, J.: Automata Studies. (AM-34). Princeton University Press (1956).

23. Nilsson, N.J.: The Quest for Artificial Intelligence: A History of Ideas and Achievements. Cambridge University Press, Cambridge (2009). https://doi.org/10.1017/CBO9780511819346.

24. Butz, M.V.: Towards Strong AI. Künstl Intell. 35, 91-101 (2021). https://doi.org/10.1007/s13218-021-00705-x.

25. Schneider, S.: Superintelligent AI and the Postbiological Cosmos Approach. In: Losch, A. (ed.) What is Life? On Earth and Beyond. pp. 178-198. Cambridge University Press, Cambridge (2017). https://doi.org/10.1017/9781316809648.011.

26. Massmann, C., Hofstetter, A.: AI-pocalypse now? Herausforderungen Künstlicher Intelligenz für Bildungssystem, Unternehmen und die Workforce der Zukunft. Digitale Bildung und Künstliche Intelligenz in Deutschland. 167-220 (2020). https://doi.org/10.1007/978-3-658-30525-3_8.

27. Wick, C.: Deep Learning. Informatik Spektrum. 40, 103-107 (2017). https://doi.org/10.1007/s00287-016-1013-2.

28. Schmitt, B.: Speciesism: an obstacle to AI and robot adoption. Mark Lett. 31, 3-6 (2020). https://doi.org/10.1007/s11002-019-09499-3.

29. Brynjolfsson, E., McAfee, A.: The Business of Artificial Intelligence. 20.

30. Zhang, R., McNeese, N.J., Freeman, G., Musick, G.: “An Ideal Human": Expectations of AI Teammates in Human-AI Teaming. Proc. ACM Hum.-Comput. Interact. 4, 1-25 (2021). https://doi.org/10.1145/3432945.

31. Adadi, A., Berrada, M.: Peeking Inside the Black-Box: A Survey on Explainable Artificial Intelligence (XAI). IEEE Access. 6, 52138-52160 (2018). https://doi.org/10.1109/ACCESS.2018.2870052.

32. Brock, J.K.-U., von Wangenheim, F.: Demystifying AI: What Digital Transformation Leaders Can Teach You about Realistic Artificial Intelligence. California Management Review. 61, 110-134 (2019). https://doi.org/10.1177/1536504219865226.

33. Woodruffe, C.: What Is Meant by a Competency? Leadership \& Org Development J. 14, 29-36 (1993). https://doi.org/10.1108/eb053651. 
34. Boyatzis, R.E.: Competencies in the 21 st century. Journal of Management Development. 27, 5-12 (2008). https://doi.org/10.1108/02621710810840730.

35. Pfeiffer, S.: Kontext und KI: Zum Potenzial der Beschäftigten für Künstliche Intelligenz und Machine-Learning. HMD. 57, 465-479 (2020). https://doi.org/10.1365/s40702-02000609-8.

36. Ehsan, U., Tambwekar, P., Chan, L., Harrison, B., Riedl, M.O.: Automated rationale generation: a technique for explainable $\mathrm{AI}$ and its effects on human perceptions. In: Proceedings of the 24th International Conference on Intelligent User Interfaces. pp. 263274. ACM, Marina del Ray California (2019). https://doi.org/10.1145/3301275.3302316.

37. Zweig, K.A.: Algorithmische Entscheidungen: Transparenz und Kontrolle. 16 (2019).

38. Yampolskiy, R.V., Spellchecker, M.S.: Artificial Intelligence Safety and Cybersecurity: a Timeline of AI Failures. 12

39. Brynjolfsson, E., Mitchell, T.: What can machine learning do? Workforce implications. Science. 358, 1530-1534 (2017). https://doi.org/10.1126/science.aap8062.

40. Zweig, K.A., Lischka, K.: Wo Maschinen irren können: Verantwortlichkeiten und Fehlerquellen in Prozessen algorithmischer Entscheidungsfindung. Arbeitspapier Algorithmenethik. (2018). https://doi.org/10.11586/2018006.

41. Van Aartrijk, M.L., Tagliola, C.P., Adriaans, P.W.: AI on the Ocean: the RoboSail Project. In: ECAI. pp. 653-657 (2002).

42. Conrad, C.S.: Künstliche Intelligenz - Die Risiken für den Datenschutz. Datenschutz Datensich. 41, 740-744 (2017). https://doi.org/10.1007/s11623-017-0870-4.

43. Gausling, T.: KI und DS-GVO im Spannungsverhältnis. Künstliche Intelligenz. 11-53 (2020). https://doi.org/10.1007/978-3-658-30506-2_2.

44. Dastin, J.: Amazon scraps secret AI recruiting tool that showed bias against women, https://www.reuters.com/article/us-amazon-com-jobs-automation-insightidUSKCN1MK08G, (2018).

45. Smuha, N.A.: The EU Approach to Ethics Guidelines for Trustworthy Artificial Intelligence. Computer Law Review International. 20, 97-106 (2019). https://doi.org/10.9785/cri-2019-200402.

46. Thiebes, S., Lins, S., Sunyaev, A.: Trustworthy artificial intelligence. Electron Markets (2020). https://doi.org/10.1007/s12525-020-00441-4.

47. Hagendorff, T.: The Ethics of AI Ethics: An Evaluation of Guidelines. Minds \& Machines. 30, 99-120 (2020). https://doi.org/10.1007/s11023-020-09517-8.

48. Baylor, A.L.: Promoting motivation with virtual agents and avatars: role of visual presence and appearance. Philosophical Transactions of the Royal Society B: Biological Sciences. (2009). https://doi.org/10.1098/rstb.2009.0148.

49. Sonnentag, S., Kuttler, I., Fritz, C.: Job stressors, emotional exhaustion, and need for recovery: A multi-source study on the benefits of psychological detachment. Journal of Vocational Behavior. 76, 355-365 (2010). https://doi.org/10.1016/j.jvb.2009.06.005. 